\title{
Investigation of metal deformation using thermography
}

\author{
by W. Oliferuk
}

Institute of Fundamental Technological Research, Polish Academy of Science, Warsaw

\begin{abstract}
Three applications of thermography are presented to investigate metal deformation. All of them are based on monitoring the temperature distribution on a metal surface during its deformation. The first application is the determination of the onset of plastic deformation on a macroscopic scale, based on the thermomechanical coupling. The second involves the use of thermography to monitor the evolution of the plastic zone and to predict the location of the necking in the sample under test. Third, thermography is used to determine the energy balance during the uniaxial tensile deformation of austenitic steel.
\end{abstract}

\section{Introduction}

Deformation processes always modify the temperature field of a material [1-3]. Immediately after the loading of elastic-plastic material the elastic deformation dominates; whereas the plastic contribution although marked in some individual grains [3] does not produce any observable macroscopic effect. In the elastic deformation stage under adiabatic conditions, the temperature of the deforming sample changes.

On the assumption of linear and isotropic material elastic behaviour, the adiabatic temperature change can be expressed by the Kelvin formula

$$
\Delta T=-\frac{\alpha \cdot T \cdot \Delta \sigma}{c_{\sigma}},
$$

where $\alpha$ is the coefficient of linear thermal expansion, T the initial absolute temperature, $\Delta \sigma$ the change in uniaxial Cauchy stress tensor, and $\mathrm{c}_{\sigma}$ the heat capacity per unit volume at constant stress.

Consequently, materials with a positive coefficient of thermal expansion reduce their temperature during adiabatic-elastic extension. The phenomenon is named thermoelastic effect.

In deformation processes there is a point where the temperature of the sample starts to increase rapidly, because a certain fraction of the mechanical energy expended in plastic deformation becomes predominant. Therefore such a temperature increase can be used to determine the onset of macroscopic plastic deformation.

The measurements of the temperature field of the sample under mechanical loading allow to investigate the non uniform and localised deformation. Such temperature measurements are also necessary to study the energy balance during the plastic deformation.

The temperature of sample surfaces was determined with a AGA Thermovision System type 680 whose operation is modified by the PTR WIN System manufactured by CEDIP/France. The PTR WIN consists of the digitiser and the PTR 9020 software running under WINDOWS. It allows to digitise a video signal into a 12 bit digital signal at the sampling frequency $1 \mathrm{MHz}$. The resulting thermal image is displayed on a computer screen. The PTR WIN System allows for continuos storing of a sequence of IR images (16 frames per second) on hard disk and for image processing (e.g. masking zones where minimum, maximum and average temperature are determined if the emissivity values for each zone are known). The temperature along an arbitrary selected line of the tested surface can also be measured. So the integration of the Thermovision System 680 with PTR WIN System resulted in a state of the art instrument. 


\section{Determination of the onset of plastic deformation on a macroscopic scale}

When certain materials such as some of austenitic steels are deformed in tensile tests, it is found that the stress-strain curve is smooth. In such a case the determination of the yield commencement is more difficult than in materials in which Luders bands take place.

The plastic deformation may occur immediately after loading in some appropriately oriented grains but the dominant deformation mode is elastic. The stress where plastic deformation or yield commencement can be observed for the first time depends both on the sensitivity of measurement and on the determination initiation of yield on a macroscopic scale. Therefore the development of micro-plastic deformation becomes a matter of convention. Usually it is assumed that plastic deformation begins when any macroscopic parameter describing the material behaviour shows detectable irregularities.

The majority of methods to determine the onset of elastic deformation is based on the stress-strain curve [4]. Three criteria for the initiation of yielding have been used: the elasticity limit, the proportionality limit, and the yield strength. The elasticity limit is the maximum stress that the material can accept without any measurable permanent strain remaining after the complete release of load. With increasing sensitivity of strain measurement, the value of the elasticity limit is reduced until it equals the true elasticity limit determined from micro strain measurements. Determination of the elasticity limit requires a tedious incremental loading-unloading test procedure. For this reason, it is often replaced by the proportionality limit.

The proportionality limit is the highest stress up to which there is proportionality to strain. It is obtained by observing the deviation from the straight-line part of the stress-strain curve.

The yield strength is the stress required to produce a small specified amount of plastic deformation. It is usually specified as a strain of $0.2 \%$.

The measurement of temperature fields on the sample under test provides a possibility to determine the onset of plastic deformation on the basis of the thermoelastic effect. Examples of dependencies $\Delta T$ and $\varepsilon$ versus $\sigma$ at a very early loading stage of specially prepared austenitic stainless steel are presented in Fig. 1 where $\Delta T$ is the average temperature of the surface of the sample's gauge part. The samples were strained on an Instron machine at a constant strain rate of $0.12 \mathrm{~min}^{-1}$.

The stress corresponding to the temperature minimum is assumed as the critical resolved stress where the plastic deformation on a macroscopic scale begins. A unique feature of the presented approach is independence of the stress value corresponding to the temperature minimum on the sensitivity of measurements.

Some investigators assume that during uniaxial adiabatic tension the plastic deformation on a macroscopic scale begins when the $\Delta T$ versus $\sigma$ relation obtained experimentally is no longer linear [5]. Such an assumption is valid only for adiabatic strain conditions. Yield stresses derived therefrom have the same uncertainty as those based on the stress-strain curve.

\section{Observation of non uniform and localised deformation}

In early stages of straining pure metals and alloys, which in the initial state have low and uniform dislocation density, exhibit deformation patterns which are essentially homogeneous on a macroscopic scale.

When the deformation of the sample is uniform its surface is isothermal. With growing strain the temperature of the sample rises. However, in the initial stages of straining the sample surface stays isothermal. Some images of the temperature distribution on the sample surface corresponding to higher values of strain show that a non uniform and localised deformation has appeared (Fig. 2).

Necking is associated with reaching an ideally plastic state. It corresponds to the maximum of the stress/strain curve $\sigma$ versus $\varepsilon$. Using the Thermovision System we can predict the location of necking. Relating the IR images or the temperature images of the 
sample under straining to the $\sigma$ versus $\varepsilon$ curve we can determine the value of $\sigma$ or $\varepsilon$ where non uniform deformation appears for given máteriäl. We can determine also an încreâse of the maximum or average temperature value of sample surface during the straining. The results for uniaxial tensile straining of austenitic stainless steel is shown in Fig. 3. On the $\sigma$ versus $\varepsilon$ curve the points are marked where the temperature images of the sample surface were obtained. The point e corresponds to the stress where non uniform deformation appears (Figs. 2 and 3 ).

\section{The investigation of the energy balance during uniaxial tensile deformation.}

When metals are cold worked, a certain fraction of the mechanical energy $e_{w}$ expended in plastic deformation is retained in the structure of material while the remaining fraction of energy is dissipated in the form of heat $q_{d}$. The retained energy is the stored energy $e_{s}$

$$
e_{w}=e_{s}+q_{d}
$$

The energy dissipated by the sample during plastic deformation is

$$
q_{d}=T_{0} s_{s}+q_{c}-e_{s e}
$$

where $s_{s}$ is configuration entropy, $q_{c}$ the heat which would have been transferred to the surroundings if the temperature of unloaded sample had returned to initial temperature, and $e_{t e}$ the energy associated with thermoelastic coupling.

Assuming that for metals $\mathrm{s}_{\mathrm{s}} \approx 0$,

$$
\begin{gathered}
q_{d}=q_{c}-e_{u} \\
e_{t}=\frac{\alpha T_{0} \sigma}{\rho}
\end{gathered}
$$

Where $\rho$ is the density of the tested metal.

The heat $q_{c}$ is determined by simulating the process of sample heating during deformation by applying electrical power $r\left(t_{1}\right)$ in such a way that the temperature increase with time $t_{1}$ during the simulation is identical with that measured during tensile testing [6]. When the straining and the simulation are performed under identical conditions, the heat $q_{c}$ in both cases is the same and is equal to

$$
q_{c}=\int_{0}^{1} r\left(t_{1}\right) d t_{1}
$$

Therefore one obtains

$$
q_{d}=\int_{0}^{t} r\left(t_{1}\right) d t_{1}+\frac{\alpha}{\rho} T_{0} \sigma .
$$

Thus

$$
e_{s}=e_{w}-\int_{0}^{i} r\left(t_{1}\right) d t_{1} \frac{\alpha}{\rho} T_{0} \sigma
$$

The energy $e_{w}$ is found from the load versus elongation curve.

A unique feature of the method is the possibility to determine the dissipated energy $q_{d}$ and stored energy $e_{s}$ as a function of $\varepsilon$ without interrupting the tensile tests and without the need of a calorimeter. Resuts of such measurments for austenitic steel are presented in Fig. 4. 


\section{Conclusions}

The temperature distribution on the surface of deformed sample can be the basis for determine the initiation of yielding on a macroscopic scale. A unique feature of the presented method is its independence of results on the measurement sensitivity.

The temperature measurements based on the IR radiation detection allow to observe the evolution of the plastic zone and to predict the location of necking in the deformed sample.

It has been shown that the contactless measurements of temperature make it possible to measure the energy balance in a metal without interrupting the deformation process.

The described methods do not exhaust the use of thermography to investigate the behaviour of metal. It can be used, for example, to determine the temperature field in a sample subjected to Taylor's test.

\section{Acknowledgements}

This work has been supported by the State Committee for Scientific Research under Grant No 7 T08A 02511.

\section{REFERENCES}

[1] TAYLOR (G. I.) - The Latent Energy Remaining in a Metal after Cold Working. Proc. Roy. Soc., 1934, p. 307-326.

[2] KLEPACZKO (J.) - Sprzężenia termomechaniczne w metalach. IFTR Reports. 1978, 11

[3] TANDON (K. N.), TANGRI (K.) - Slip Sources in the Surface Layers of Polycrystalline Fe3 Pct Si in Early Stages of Deformation. Metal. Trans., 1975, 6A, p. 809-813.

[4] DIETER (G. E.) - Mechanical Behaviour of Materials under Tension. Metals Handbook, Mechanical Testing, 1985, 8, p. 20-28.

[5] GABRYSZEWSKI (Z.) and PINDUR (B.) - Ocena powierzchni plastyczności materiałów anizotropowych nieliniowych metodą sprzężenia termo-mechanicznego. Mech. Teoret. i Stos., $1985,23,2$, p. 291-305.

[6] OLIFERUK (W.), CEWI¥TNICKI (W. A.) and GRABSKI (M. W.) - Effect of the Grain size on the Rate of Energy Storage during the Tensile Deformation of an Austenitic Steel. Mater. Sci. Eng., 1995, A197, p. 49-58.

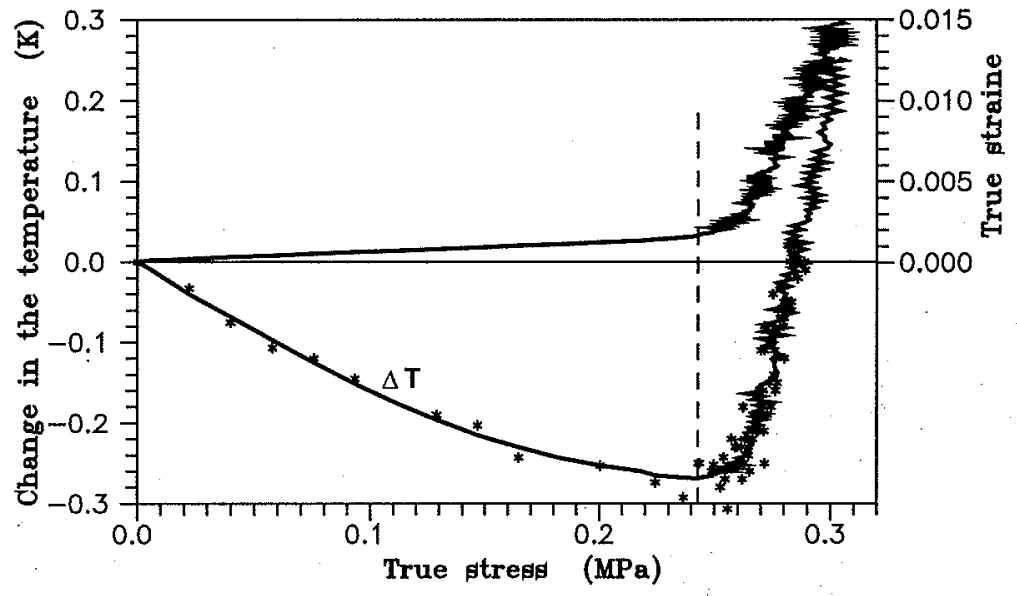

Fig.1. Dependencies of strain and change of sample temperature on stress in an early stage of austenitic steel tension 
http://dx.doi.org/10.21611/qirt.1998.021
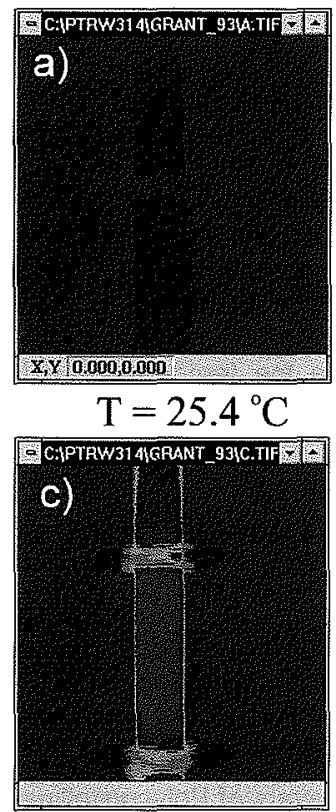

$$
\mathrm{T}=35.4{ }^{\circ} \mathrm{C}
$$
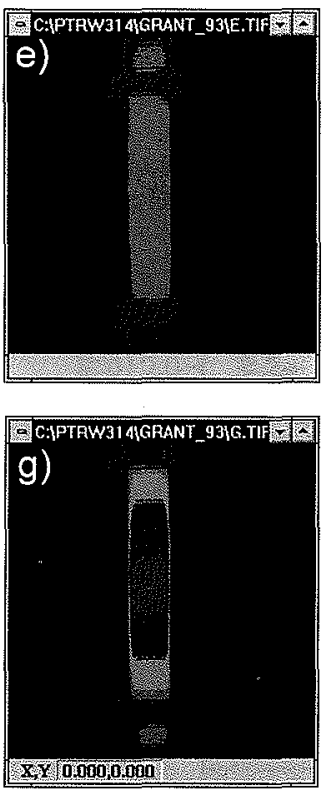
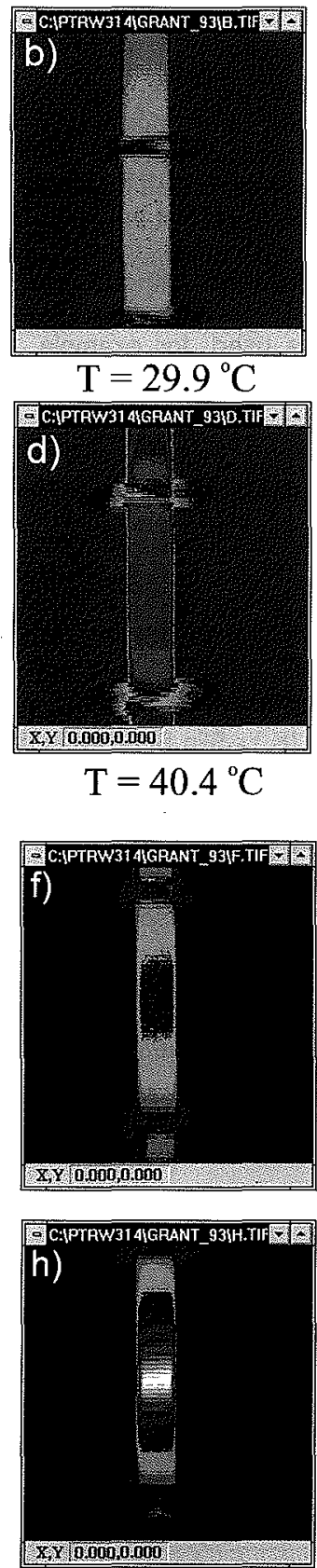
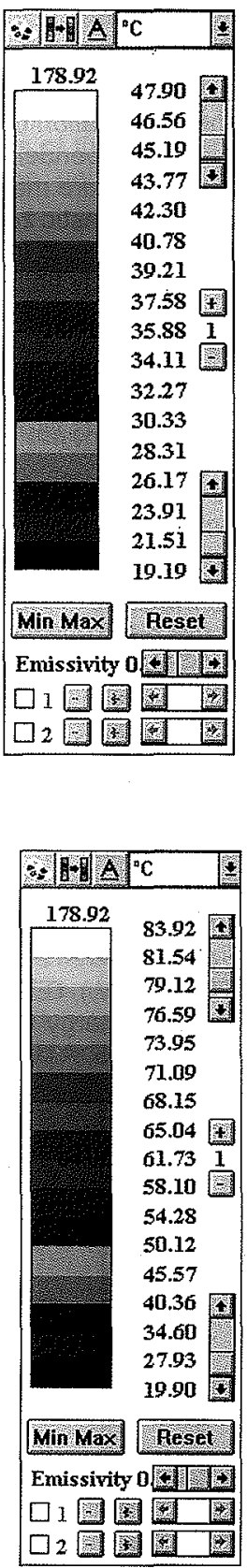

Fig. 2. Temperature distribution on the surface of an austenitic steel sample during uniaxial tension. a) $-\varepsilon=0.05, \mathrm{~b})-\varepsilon=0.14, \mathrm{c})-\varepsilon=0.22$, d) $-\varepsilon=0.30, e$ ) $-\varepsilon=0.36$, f) $-\varepsilon=0.43$, g) $-\varepsilon=0.47$, h) $-\varepsilon=0.51$ 
http://dx.doi.org/10.21611/qirt.1998.021

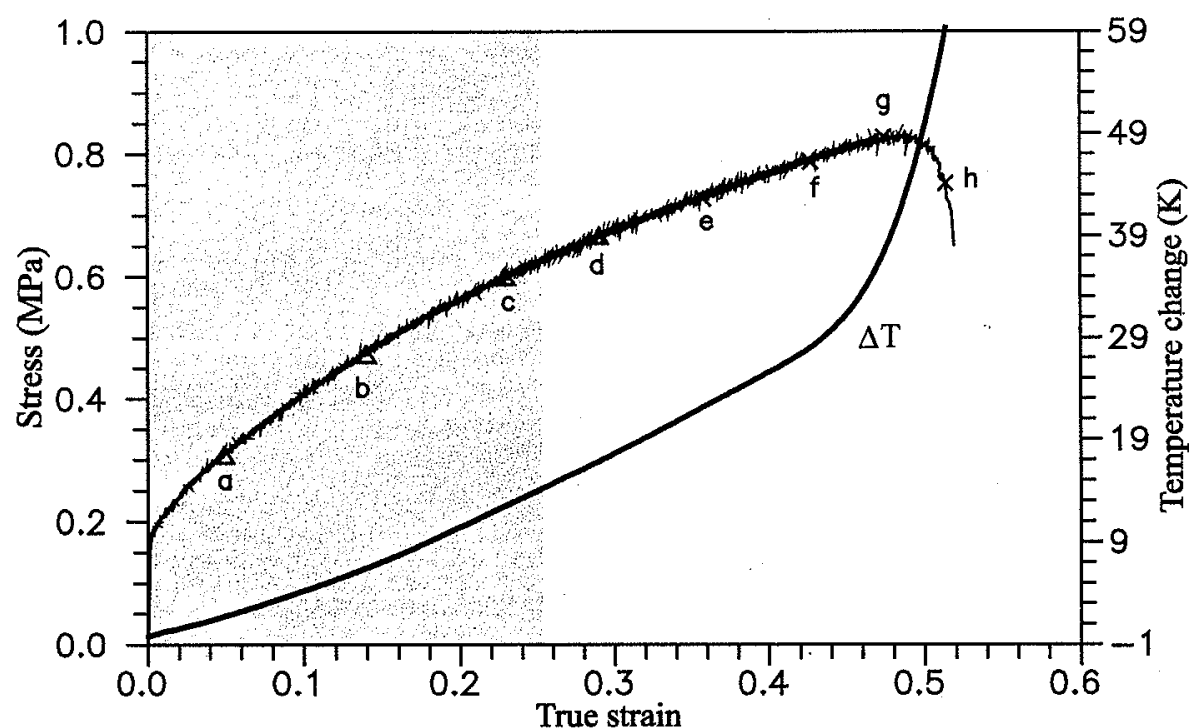

Fig. 3. Stress and increase of sample temperature as function true strain obtained during tension of austenitic steel. Points indicate where temperature images were taken.

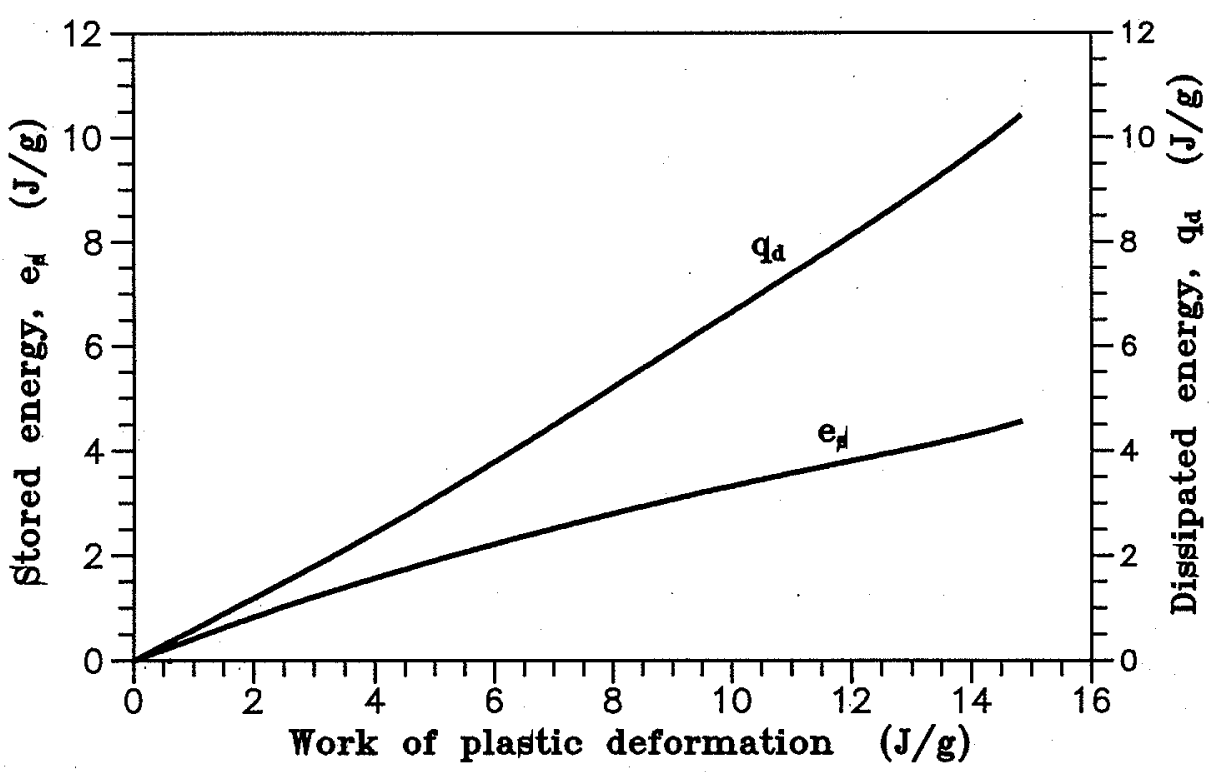

Fig. 4. Stored and dissipated energies depending on plastic deformation work 